\title{
ON QUOTIENTS OF HOLOMORPHIC FUNCTIONS IN THE DISC WITH BOUNDARY REGULARITY CONDITIONS
}

\author{
JOAQUIN M. ORTEGA
}

Abstract

In this paper we give characterizations of those holomorphic functions in the unit disc in the complex plane that can be written as a quotient of functions in $A(D), A^{\infty}(D)$ or $\Lambda_{1}(D)$ with a nonvanishing denominator in $D$. As a consequence we prove that if $f \in \Lambda_{1}(D)$ does not vanish in $D$, then there exists $g \in \Lambda_{1}(D)$ which has the same zero set as $f$ in $\bar{D}$ and such that $f g \in A^{\infty}(D)$.

\section{Introduction and statement of results}

Let $D$ denote the unit disc in the complex plane and $T$ its boundary. We denote by $A(D)$ the Banach algebra of all continuous functions on $\bar{D}$, holomorphic in $D$, and by $A^{\infty}(D)$ the Frechet algebra of all holomorphic functions in $D$ such that all its derivatives extend continuously to $\bar{D}$. We will also consider the Banach algebra $\Lambda_{1}(D)$ consisting of all holomorphic functions in $D$ satisfying a Lipschitz conditions of order one.

In this paper we are interested in the characterization of those holomorphic functions in $D$ that can be written as a quotient of functions in $A(D), A^{\infty}(D)$ or $\Lambda_{1}(D)$, respectively, with a nonvanishing denominator in $D$.

The corresponding real-variable problems are very simple. For example, every continuous (resp. $C^{\infty}$ ) function in an open set $V$ of $\mathbf{R}^{n}$ is a quotient of two continuous (resp. $C^{\infty}$ ) functions in $R^{n}$ vanishing exactly on $R^{n} \backslash V$ (see [6]). On the other hand, this kind of problems has also been treated in complex analysis, the most well known one being the result of $F$. and $R$. Nevanlinna which characterizes the quotients of bounded holomorphic functions in $D$ as the functions in the class $N$, i. e.

$$
\sup _{r} \int_{0}^{2 \pi} \log ^{+}\left|f\left(r e^{i t}\right)\right| d t<\infty .
$$

Supported by grant PB85-0374 of the CAICYT. Ministerio de Educación y Ciencia. Spain. 
(see also [11] for another problem of this type).

Before stating our main results we will recall some well-known notions and introduce some notations. Each function $f$ in $N$ has an unique factorization $f=B S F$, where $B$ is the Blaschke product with the same zero sequence as $f, F$ is the outer function with boundary absolute value $|F|=|f|$ and $S$ is a singular function

$$
S(z)=\exp \left\{-\int_{0}^{2 \pi} \frac{e^{i t}+z}{e^{i t}-z} d \mu(t)\right\}
$$

for some signed measure $\mu$. The descomposition of $d \mu$ in its positive and negative parts gives $S=S_{1} / S_{2}$, with $S_{1}, S_{2}$ singular inner functions. The $S$ mirnov class $N^{+}$is the subclass of $N$ defined by the condition $S_{2}=1$. We will use the fact that $f \in N^{+}$if and only if the family $\log ^{+}\left|f_{r}\right|$, where $f_{r}\left(e^{i \theta}\right)=f\left(r e^{i \theta}\right)$, has uniformly absolutely continuous integrals.. From this it easily follows that $N^{+}$is a linear space (see [3, chapter 2]) as a general reference on $N$ and $N^{+}$).

If $f \in N$ is as above we will write

$$
\operatorname{sing}(f)=E \cup \operatorname{supp} \mu,
$$

where $E$ is the set of accumulation points of the zeros of $f$ and supp $\mu$ is the closed support of $\mu$. Then $B$ and $S$ extend analytically to $T \backslash \operatorname{sing}(f)$ and are not zero there (see $[4$, p.68]).

Now we state our first result:

Theorem 1. An holomorphic function $f$ in $D$ is the quotient of two functions $f_{1}, f_{2} \in A(D), f_{2} \neq 0$ in $D$ if and only if the following two conditions hold:

(a) $f \in N$.

(b) There is a closed set $M$ of Lebesque measure zero such that:

(b.1) $f$ extends continuously to $T \backslash M$.

(b.2) sing $\left(S_{2}\right) \subset M$, where $f=B F S_{1} / S_{2}$ is the factorization of $f$.

In this case, $f_{2}$ can be chosen to vanish exactly on $M$ and outer if and only if $f \in N^{+}$.

To state our other main theorems we need the notion of Carleson set. These are the closed sets $M \subset T$ such that

$$
\int_{T} \log d\left(e^{r \theta}, M\right) d \theta>-\infty
$$

where $d\left(e^{i \theta}, M\right)$ denotes the distance from $e^{i \theta}$ to $M$ (and so, obviously, subsets and finite unions of Carleson sets are Carleson sets, too). These sets are precisely the zero boundary sets of functions in $A^{\infty}(D)$ and $\Lambda_{1}(D)$ (see $[8]$ ). Finally, we say that $f \in A^{\infty}(D)$ is flat at a closed set $E \subset T$ if $f^{(n)}(z)=0$ for all $n$ and $z \in E$. 
Theorem 2. For an holomorphic function $f$ in $D$ to be the quotient of two functions $f_{1}, f_{2} \in A^{\infty}(D), f_{2} \neq 0$ in $D$ it is necessary and sufficient that:

(a) $f^{(n)} \in N$ for all $n$, and if $f=B F S_{1} / S_{2}$ is its factorization then $\left(S_{2} f\right)^{(n)} \in N^{+}$for all $n$.

(b) There is a Carleson set $M$ such that:

(b.1) There are a function $g$, satisfying a Lipschitz condition of order 1 in $T$, with $\log g \in L^{1}(T)$, positive outside $M$, and for each $n$ an integer $q_{n}$ such that

$$
\left|f^{(n)}(z)\right|^{1 / q_{n}}=0\left(g(z)^{-1}\right), z \in T \backslash M .
$$

(b.2) $\operatorname{sing}\left(S_{2}\right) \subset M$.

In this case, $f_{2}$ can be chosen flat exactly on $M$ and outer if and only if $f \in N^{+}$.

Theorem 3. An holomorphic function $f$ in $D$ is the quotient of two functions $f_{1}, f_{2} \in \Lambda_{1}(D), f_{2} \neq 0$ in $D$ if and only if the conditions $(a)$ and $(b)$ of Theorem 2 hold for $n=0$ and $n=1$. In this case, $f_{2}$ can always be chosen in $A^{\infty}(D)$ and flat exactly on $M$ and outer if and only $f \in N^{+}$.

In condition (b) of Theorem 2 and $3, f^{(n)}$ is to be understood as the almost everywhere defined boundary value of the function $f^{(n)} \in N$. It is not difficult to see directly that conditions (a) and (b) imply in fact that $f^{(n)}$ has a continuous extension to $T \backslash M$ (for all $n$ in Theorem 2 and $n=0$ in Theorem 3). We point out however that, contrary to what one might expect in analogy with Theorem I, condition (b.1) cannot be replaced by the weaker assumption that $f^{(n)}$ extends continuously to $T \backslash M$ for all $n$ (see Remark 2 in section 3 ).

Acknowledgement. I wish to thank J. Bruna for several remarks and helpful conversations about this work.

\section{Proof of Theorem 1}

Lemma 1. Assume $f \in N$ and that there is a closed set $M$ such that $f$ extends continuously to $T \backslash M$. Let $K=\{z \in T \backslash M: f(z)=0\}$. Then $\operatorname{sing}(f) \subset M \cup K$ and the outer part $F$ of $f$ also extends continuously to $T \backslash M$.

Proof: This is well known when $M=\phi$ (see [4,p.69]) and the same proof applies in this general case.

Lemma 2. Let $\varphi$ be a non-negative continuous function in $(a, b)$ such that $\log \varphi$ is integrable. Then there exists a $C^{\infty}$ function $\psi$ in $(a, b)$ such that $\psi \geq 1, \psi \geq \varphi$ and

$$
\int_{a}^{b} \log \psi(t) d t \leq 4 \int_{a}^{b} \log ^{+} \varphi(t) d t .
$$


Proof: We consider a partition of $(a, b)$ in intervals

$$
\left[a+\frac{b-a}{2^{n+1}}, a+\frac{b-a}{2^{n}}\right],\left[b-\frac{b-a}{2^{n}}, b-\frac{b-a}{2^{n+1}}\right), \quad n \geq 1 .
$$

Let $[c, d)$ be one of these intervals. Let $s$ be a step function in $[c, d)$ such that

$$
\log ^{+} \varphi \leq s, \int_{c}^{d} s \leq 2 \int_{c}^{d} \log ^{+} \varphi \text {. }
$$

Doing the same in every interval we obtain a subdivision of $(a, b)$ in intervals $\left[\lambda_{n}, \lambda_{n+1}\right)$ and a function $g$, equal to some constant $c_{n}$ on $\left\{\lambda_{n}, \lambda_{n+1}\right\}$ such that

$$
\log ^{+} \varphi \leq g, \int_{a}^{b} g \leq 2 \int_{a}^{b} \log ^{+} \varphi
$$

We can also assume that $c_{n} \geq 0$ and $c_{n} \neq c_{n+1}$. Let $\left[\lambda_{n}, \lambda_{n+1}\right)$ and $\left(\lambda_{n+1}, \lambda_{n+2}\right)$ be two contiguous intervals and let $\mu_{n}$ and $\mu_{n+1}$ their respective middle points. It is easy now to construct a function $h$ on $\left[\mu_{n}, \mu_{n+1}\right]$ which verifies:

1) $h(x) \geq c_{n}$ for $x \in\left[\mu_{n}, \lambda_{n+1}\right]$ and $h(x) \geq c_{n+1}$ for $x \in\left[\lambda_{n+1}, \mu_{n+1}\right]$.

2) $h\left(\mu_{i}\right)=2 c_{i}, i=n, n+1$.

3) $h$ is $C^{\infty}$ and all its derivatives vanish at $\mu_{n}$ and $\mu_{n+1}$.

4) $\int_{\mu_{n}}^{\mu_{n+1}} h \leq 2 \int_{\mu_{n}}^{\mu_{n+1}} g$.

Doing the same with all intervals we obtain a $C^{\infty}$ function in $(a, b)$, which we continue denoting by $h$, such that $h \geq g$ and

$$
\int_{a}^{b} h \leq 2 \int_{a}^{b} g
$$

Then $\psi=e^{k}$ satisfies all required conditions.

Proof of Theorem 1: The necessity of the conditions is clear in all the cases, using Lemma 1 for part (b.2). Assume now first that $f \in N^{+}$and extends continuously to $T \backslash M$. Let $f=B S F$. Applying Lemma 2 to $\varphi=|f|$ in each complementary interval of $T \backslash M$ we obtain a $C^{\infty}$ function $\varphi_{1}$ in $T \backslash M$, $\varphi_{1} \geq 1, \varphi_{1} \geq \varphi$ with $\log \varphi_{1} \in L^{1}(T)$. Now we consider the outer functions $F_{1}, F_{2}$ with boundary values $\left|F_{1}\right|=\varphi / \varphi_{1},\left|F_{2}\right|=1 / \varphi_{1}$, respectively, so that $\left|F_{1}(z)\right|,\left|F_{2}(z)\right| \leq 1$ and $F_{1}=F F_{2}$. The function $F_{2}$ extends continuously to $T \backslash M$ because $\log \varphi_{1}$ is $C^{\infty}$ off $M$ by Lemma 1 and hence $F_{1}=F F_{2}$ also extends continuously to $T \backslash M$. Let now $G$ be an outer function in $A(D)$ vanishing exactly on $M$. Then $F_{1} G, F_{2} G$ are outer functions in $A(D), F_{2} G=0$ exactly on $M$ and $F_{1} G=0$ on $M \cup K$ where $K$ is as in Lemma 1. Now

$$
f=\frac{B S F_{1} G}{F_{2} G} .
$$


Since by Lemma 1 , sing $(B S) \subset M \cup K$ and $F_{1} G$ vanishes there, it follows that $f_{1}=B S F_{1} G \in A(D)$. This proves the theorem when $F \in N^{+}$. If $f$ is just in $N$ and $f=B F S_{1} / S_{2}$ with $\operatorname{sing}\left(S_{2}\right) \subset M$ repeating the proof we end up with

$$
f=\frac{B S_{1} F_{1} G}{S_{2} F_{2} G}
$$

and also $S_{2} F_{2} G \in A(D)$ because $\operatorname{sing}\left(S_{2}\right) \subset M$ and $F_{2} G$ vanishes on $M$.

\section{Proof of Theorem 2}

In order to prove Theorems 2 and 3 we will follow the method used by B.A. Taylor and D.L. Williams in $[\mathbf{1 1}\}$ to obtain such a representation for certain Blaschke products. As in [11], we say that a function defined in (a, b) satisfies a weak Lipschitz condition if

$$
|\varphi(t+\Delta t)-\varphi(t)| \leq 2|\Delta t||\varphi(t)|^{2}
$$

for $|\Delta t| \leq 1 / 2|\varphi(t)|^{-1}$. We will use the following three lemmas (see [11] for Lemmas 3 and 4 and [10] for Lemma 5 ):

Lemma 3. Let $\varphi$ be a real-valued in (a, b) which satisfies a weak Lipschitz condition, $\varphi \geq 2$, and

$$
|\varphi(t)| \geq(\min \{|t-a|,|t-b|))^{-1} .
$$

Then there exists a real-valued $C^{\infty}$ function $h$ in $(a, b)$ such that $h \geq 2$, $\varphi-2 \leq h \leq \varphi+2$ and $\left|h^{(n)}\right| \leq c_{n} \varphi^{3 n}$ for some constant $c_{n}$ and all $n$.

Lemma 4. Let $g$ be a non-negative integrable furction on $[-\pi, \pi]$. There exists a non-negative $C^{\infty}$ function $w(x)$, defined for $x \geq 0$, which verifies:

(a) $x^{-1} w(x) \rightarrow+\infty$ as $x \rightarrow+\infty$.

(b) $\int_{-\pi}^{\pi} w(g(t)) d t<+\infty$.

(c) For each $n$ there exists a constant $c_{n}$ such that

$$
\left|w^{(n)}(x)\right| \leq c_{n}\left(1+x^{2}\right) .
$$

Lemma 5. Let $f \in A^{\infty}(D)$, let $f=B S F$ and let $K=\left\{z \in T: f^{(n)}(z)=0\right.$ for all $n\}$. Then $\sin g(f) \subset K$, (so that sing $(f)$ is a Carleson set), $F \in A^{\infty}(D)$ and $F$ is fat on $K$. More generally, if $f \in A^{\infty}(D)$ and $S_{0}$ is a singular inner function dividing $f$ (i. e. $S / S_{0}$ is bounded), then $f / S_{0} \in A^{\infty}(D)$. Conversely, if $S$ is a singular inner function and $F \in A^{\infty}(D)$ is flat on $\operatorname{sing}(S)$ then $F S \in A^{\infty}(D)$.

Proof of Theorem 2: Let $f=f_{1} / f_{2}$, with $f_{1}, f_{2} \in A^{\infty}(D), f_{2} \neq 0$ in $D$, and let $M$ be the set of zeros of $f_{2}$, a Carleson set in $T$. It is obvious that 
$f^{(n)} \in N$ for all $n$ and that $f^{(n)} f_{2}^{n+1} \in A^{\infty}(D)$, and hence (b.1) holds with $q_{n}=n+1$ and $g=\left|f_{2}\right|$. It remains to prove that $\operatorname{sing}\left(S_{2}\right) \subset M$ and that $\left(S_{2} f\right)^{(n)} \in N^{+}$. Let $d \nu_{1}, d \nu_{2}$ be the measures corresponding to the singular parts of $f_{1}, f_{2}$ respectively and $d \mu_{1}, d \mu_{2}$ the ones corresponding to $S_{1}$ and $S_{2}$. Then $\mu_{1}=\left(\nu_{1}-\nu_{2}\right)_{+} \leq \nu_{1}, \mu_{1}-\mu_{2}=\nu_{1}-\nu_{2}$, that is, $\nu_{i}=\mu_{i}+\nu, i=1,2$ for some positive measure $\nu$. Therefore, $\operatorname{sing}\left(S_{2}\right) \subset \operatorname{supp} \mu_{2} \subset M$, by Lemma 5. Also, the singular inner function $S_{0}$ corresponding to $d \nu$ divides both $f_{1}, f_{2}$ and so by Lemma $5, f_{1} / S_{0}$ and $f_{2} / S_{0}$ are again in $A^{\infty}(D)$. This means that replacing $f_{1}$ by $f_{1} / S_{0}$ and $f_{2}$ by $f_{2} / S_{0}$ we can assume that $S_{2}$ is the singular inner part of $f_{2}$. Then $S_{2} f=f_{1} / F_{2}$, where $F_{2}$ is the outer part of $f_{2}$, which is in $A^{\infty}(D)$ by Lemma 5 , and now it is clear that $\left(S_{2} f\right)^{(n)} \in N^{+}$for all $n$.

Assume now that $f \in N^{+}$satisfies (a), i. e. $f^{(n)} \in N^{+}$, for every $n$, and (b). Without loss of generality we can assume that the Lipschitz constant for $g$ is 1 , that $|g| \leq 1 / 2$ and, multiplying $g$ by a function in $A^{\infty}(D)$ vanishing on $M$, that $g$ is zero on $M$. Then the hypothesis of Lemma 3 hold for $\varphi=1 / g$ in each complementary interval of $M$ in $T$ (if $|\Delta t| \leq 1 / 2 \varphi(t)^{-1}=|g(t)| / 2$ then $g(t+\Delta t) \geq g(t) / 2$ and hence

$$
\left.|\varphi(t+\Delta t)-\varphi(t)|=\frac{|g(t+\Delta t)-g(t)|}{|g(t)||g(t+\Delta t)|} \leq \frac{|\Delta t|}{\frac{1}{2}|g(t)|^{2}}=2|\Delta t||\varphi(t)|^{2}\right) .
$$

If $h$ is the function given by Lemma 3 , then $h_{1}=h+2$ is a real valued $C^{\infty}$ function in $T \backslash M$ such that $h_{1} \geq 4,1 / g \leq h_{1} \leq 3 / g$ and for every $n$ there exists $c_{n}$ such that

$$
\left|h_{1}^{(n)}\right| \leq c_{n} g^{-3 n}
$$

In particular $h_{1}$ has an integrable logarithm. Let now $w$ be a function as in Lemma 4, with $g=\log h_{1}$. We consider the outer function $F=\exp G$ with

$$
G(z)=-\frac{1}{2 \pi} \int_{0}^{2 \pi} \frac{e^{i t}+z}{e^{i t}-z} w\left(\log h_{1}(t)\right) d t .
$$

Notice that $F \in C^{\infty}(\bar{D} \backslash M)$ and it is not zero on $\bar{D} \backslash M$.

We will prove now that all the derivates of $F$ and $f F$ are bounded and tend to zero at any point of $M$, which will finish the proof of the theorem in case $f \in N^{+}$.

First we estimate $|F|$ on $T \backslash M$. Since $w(x) / x \rightarrow+\infty$ as $x \rightarrow+\infty$ we can write

$$
|F(z)|=h_{1}(z)^{-\mu(z)} \leq(3 / g(z))^{-\mu(z)}
$$

with $\mu(z) \rightarrow+\infty$ as $z \in T$ approaches $z_{0} \in M$. We estimate now $G^{(n)}$ following a method similar to the one in Carleson [2] in the form expressed by the following Lemma (see Lemma 2.3 in $[\mathbf{1}]$ ): 
Lemma 6. Let $\Psi$ be a function of class $C^{n+1}$ in some arc $\left.J=\mid a, b\right]$ of $T$, let $c$ be the middle point of $J$ and let $A(z)$ be defined by

$$
A(z)=\int_{0}^{b} \frac{e^{i t}}{\left(e^{i t}-z\right)^{n+1}} \Psi\left(e^{i t}\right) d t \quad, \quad z \in D .
$$

If $\Psi^{0}=\Psi, \Psi_{k}\left(e^{i t}\right)=e^{-i t} \frac{d}{d t} \Psi_{k-1}\left(e^{i t}\right), k=1, \ldots, n+1$ and

$M_{k}=\max \left\{\left|\Psi_{k}\left(e^{i t}\right)\right|, a \leq t \leq b\right\}$, then for $z=r e^{i c}, 0 \leq r<1$

$$
|A(z)| \leq \operatorname{const}\left(\sum_{k=0}^{n-1} \frac{M_{k}}{\left|z-e^{i a}\right|^{n-k}}+M_{n}+|J| M_{n+1}\right) .
$$

Let $z \in T \backslash M$. In

$$
\left|G^{(n)}(r z)\right|=\frac{n !}{\pi}\left|\int_{-\pi}^{\pi} \frac{e^{i t}}{\left(e^{i t}-r z\right)^{n+1}} w\left(\log h_{1}(t)\right) d t\right|
$$

we break the integral into two parts corresponding to the interval of center $z$ and radious $\frac{1}{2}|g(z)|$, which is less than $\frac{1}{2} d(z, M)$, and its complementary. In the second integral, $\left|e^{i t}-r z\right| \geq c|g(z)|$ and so this integral is bounded by const $|g(z)|^{-n-1}$. In the first integral we use the bounds for $h_{1}$ and its derivatives, and those of $w$,obtaining with the notations of Lemma 6 ,

$$
M_{n} \leq c \max \left\{\left|g\left(e^{i t}\right)\right|^{-p_{n}}, a \leq t \leq b\right\}
$$

for some integer $p_{n}$.

Using the fact that $g(z+\Delta z) \geq \frac{1}{2} g(z)$ for $\Delta z<\frac{1}{2} g(z)$ we see that $M_{n} \leq$ const $g(z)^{-p_{n}}$ and so we conclude that

$$
\left|G^{(n)}(r z)\right| \leq \text { const } g(z)^{-p_{n}}
$$

for some integers $p_{n}$. From (1) and (2) it follows that

$$
\left|F^{(n)}(z)\right| \leq \operatorname{const}\left(\frac{3}{g(z)}\right)^{-\mu(z)} g(z)^{-q_{n}} \quad, z \in T \backslash M,
$$

for some integers $q_{n}$. Now, by the hypothesis (b.1) on $f,(f F)^{(n)}$ will also satisfy this bound. This implies that $(f F)^{(n)}$ and $F^{(n)}$ are bounded and tend to zero at any point of $M$ along $T \backslash M$, because $\mu(z) \rightarrow+\infty$.

The proof will be finished if we show that $(f F)^{(n)}$ and $F^{(n)}$ belong to $N^{+}$ for any $n$. Since $f^{(n)} \in N^{+}$for all $n$ by hypothesis, it is enough to prove that any derivate of $G$ belongs to $N^{+}$. But this follows from (2), because $\log ^{+}\left|G_{r}^{(n)}\right|$ is uniformly integrable being dominated by $\log ^{+} 1 / g$. 
This ends the proof of the sufficiency part of Theorem 2 in case $f \in N^{+}$. Assume now that $f$ satisfies (a) and (b) and $\operatorname{sing}\left(S_{2}\right) \subset M$. This later fact clearly implies that for some constanst $p_{n}$

$$
\left|S_{2}^{(n)}(z)\right| \leq \text { const } d(z, N)^{-p_{n}} \leq \text { const } g(z)^{-p_{n}}
$$

(recall that we can assume $g(z) \leq d(z, N)$ ). Hence $f S_{2}$ is a $N^{+}$function to which we can apply what we have already proved: $S_{2} f=S_{2} f F / F$ with $F$ flat on $M$. By the last part of Lemma $5, S_{2} F \in A^{\infty}(D)$ and the proof is finished.

Remark 1. A particular case of a function which verifies the condition of Theorem 3 was considered by B.A. Taylor and D.L. Williams $(\{1])$. It is a Blaschke product such that if $E$ is its set of zeros then

$$
\int \log d\left(e^{i \theta}, E\right) d \theta>-\infty
$$

In this case, the function $d\left(e^{i t}, E\right)$ plays the role of the function $g$ in condition (b.1) and $M=\bar{E} \cap T$.

Remark 2. As already said, condition (b.1) in Theorem 2 cannot be replaced by the weaker assumption that $f^{(n)}$ extends continuously to $T \backslash M$ for all $n$. Consider for instance a Blaschke product whose zero set

$$
E=\left\{r_{n} e^{i \theta_{n}}\right\}_{n \geq 1}
$$

satisfies:

(a) $\sum_{n=1}^{\infty}\left(1-r_{n}\right)^{\alpha}<+\infty$ for every $\alpha>0$.

(b) $E$ has $\{1\}$ as unique acumulation point.

(c) The set $\{1\} \cup\left\{e^{i \theta_{n}}\right\}_{n \geq 1}$ is not a Carieson set.

Every Blaschke product that satisfies (a) has derivatives $B^{(n)} \in H^{p}$ for some $p=p(n)$ (see $[5]$ ), and then $B^{(n)} \in N^{+}$. Trivially $B$ extends analitically to $T-\{1\}$, yet $B$ cannot be expresed as a quotient $f_{1} / f_{2}$ with $f_{1}, f_{2} \in A^{\infty}(B)$, because $E$, that verifies (b) and (c), is not the zero set of any function in $A^{\infty}(D)$ (see $[7]$ ).

\section{Proof of Theorem 3}

For the proof of Theorem 3 we have to use instead of Lemma 5 the following Lemma (see [9]):

Lemma 7. Let $f=B S F \in \Lambda_{1}(D)$ and $K=\{z \in T: f(z)=0\}$. Then $\operatorname{sing}(f) \subset K$, so that sing $(f)$ is a Carleson set, and $F \in \Lambda_{1}(D)$. More gener. ally, if $S_{0}$ is a singular inner function dividing $f\left(i . e . S / S_{0}\right.$ is bounded), then $f / S_{0}$ is in $\Lambda_{1}(D)$.

Proof of Theorem 3: Let $f=f_{1} / f_{2}, f_{1}, f_{2} \in \Lambda_{1}(D), f_{2} \neq 0$ in $D$ and let $M$ be the set of zeros of $f_{2}$ wich is a Carleson set in $T$. It is clear that, $f, f^{t} \in N$ 
and, as before, condition (b.1) is satisfied with $g=\left|f_{2}\right|, q_{0}=1, q_{1}=2$. With the same notations as in the proof of Theorem 2, in this case we have now, by Lemma 7 , that $f_{1} / S_{0}$ and $f_{2} / S_{0}$ are again in $\Lambda_{1}(D)$. Hence we can also write $f=g_{1} / g_{2}$ with $g_{1}, g_{2}$ in $\Lambda_{1}(D)$ and now $S_{2}$ is also the singular inner part of $g_{2}$. Then $S_{2} f=g_{1} / F_{2}$ with $F_{2} \in \Lambda_{1}(D)$. Now it is clear that $\left(S_{2} f\right)^{\prime} \in N^{+}$.

Assume now that $f_{j} f^{\prime} \in N^{+}$and that (b.1) holds. We repeat the construction of Theorem 2, thus obtaining an $F \in A^{\circ}(D)$ flat on $M$. We must check now that $f F \in \Lambda_{1}(D)$, that is, $(f F)^{\prime} \in H^{\infty}$. This is, as before, consequence of the fact that $(f F)^{\prime}=f^{\prime} F+f F^{\prime}$ belongs to $N^{+}$and that it is bounded on $T / M$. If $f \in N$ satisfies (a) and (b) then the above case applies to $S_{2} f$, so that $S_{2} f=f_{1} / f_{2}$ with $f_{2} \in A^{\infty}(D)$ and flat on $M$. Now $S_{2} f_{2} \in A^{\infty}(D)$ by Lemma 5 and we are done.

Corollary. If $f \in \Lambda_{1}(D)$ and $f \neq 0$ in $D$, there exists $g \in \Lambda_{1}(D)$ which has the same zero set as $f$ in $\bar{D}$ and such that $f g \in A^{\infty}(D)$.

Proof: Apply the theorem to $1 / f$.

\section{References}

1. J. BRUNA, Boundary interpolation sets for holomorphic functions smooth to the boundary and BMO, Transactions of the Amer. Math. Soc. 264 (2) (1981), 393-404.

2. L. CARLESON, Sets of uniqueness for functions regular in the unit circle, Acta Math. 87 (1952), 325-345.

3. P.L. DUREN, "Theory of $H^{p}$ Spaces," Academic Press, 1970.

4. K. HOFFMAN, "Banach Spaces of analytic functions," Prentice-Hall, 1962.

5. C.N. LINDEN, $H^{p}$-derivatives of Blaschke products, Michigan Math. J. 23 (1976), 43-51.

6. J. MUÑOZ AND J. MA. ORTEGA, Sobre las álgebras localmente convexas, Collectonea Mathematica XX (2) (1969), 127-149.

7. J.O. Nelson, A characterization of zero sets for $A^{\infty}$, Michigan Math. J. 18 (1971), 142-147.

8. W.P. NOVINGER, Holomorphic functions with infinitely differentiable boundary values, Mlinois $J$. Math. 15 (1971), 80-90.

9. N.A. SIROKOV, Ideals and factorization in algebras of analytic functions that are smooth up to the boundary, Proceedings of the Steklov Inst. of Math. 4 (1979), 205-233.

10. B.A. TAYLOR AND D.L. WILLIAMS, Ideals in rings of analytic functions with smooth boundary values, Canad. J. Math. XXII (6) (1970), 1266-1283. 
11. B.A. TAYlor AND D.L. Williams, Zeroes of Lipschitz functions analytic in the unit disc, Michigan Math. J. 18 (1971), 129-139.

Departament de Matemàtiques Universitat Autonoma de Barcelona 08193 Bellaterra Barcelona, SPAIN.

Rebut el 19 de Novembre de 1987 